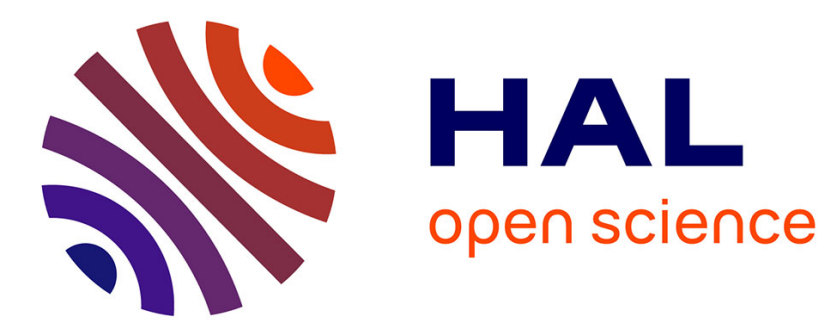

\title{
Submillimetre-wave spectrum of diacetylene and diacetylene-d2
}

Luca Bizzocchi, Claudio Degli Esposti, Luca Dore

\section{To cite this version:}

Luca Bizzocchi, Claudio Degli Esposti, Luca Dore. Submillimetre-wave spectrum of diacetylene and diacetylene-d2. Molecular Physics, 2010, pp.1. 10.1080/00268976.2010.482066 . hal-00600120

\section{HAL Id: hal-00600120 \\ https://hal.science/hal-00600120}

Submitted on 14 Jun 2011

HAL is a multi-disciplinary open access archive for the deposit and dissemination of scientific research documents, whether they are published or not. The documents may come from teaching and research institutions in France or abroad, or from public or private research centers.
L'archive ouverte pluridisciplinaire HAL, est destinée au dépôt et à la diffusion de documents scientifiques de niveau recherche, publiés ou non, émanant des établissements d'enseignement et de recherche français ou étrangers, des laboratoires publics ou privés. 


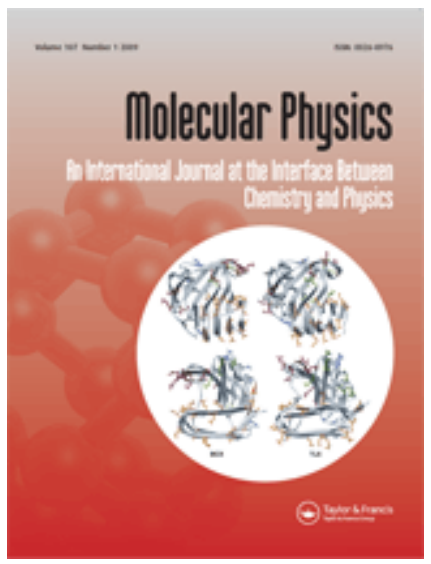

\section{Submillimetre-wave spectrum of diacetylene and diacetylene-d2}

\begin{tabular}{|r|l|}
\hline Journal: & Molecular Physics \\
\hline Manuscript ID: & TMPH-2010-0040.R1 \\
\hline Manuscript Type: & $\begin{array}{l}\text { Special Issue Paper -2 HRMS Stabia 09/ High Resolution Molecular } \\
\text { Spectroscopy }\end{array}$ \\
\hline Author: & 11-Mar-2010 \\
\hline $\begin{aligned} \text { Complete List of Authors: } \\
\text { Kubmitted by the } \\
\text { Degli Esposti, Claudio; University of Bologna, Dipartimento di } \\
\text { Chimica "G. Ciamician" } \\
\text { Dore, Luca; University of Bologna, Dipartimento di Chimica "G. } \\
\text { Ciamician" }\end{aligned}$ \\
\hline \hline & $\begin{array}{l}\text { Vibration rotation bands, Diacetylene, Hot bands, Pyrolysis, } \\
\text { Resonances }\end{array}$ \\
\hline \hline $\begin{array}{l}\text { Note: The following files were submitted by the author for peer review, but cannot be converted } \\
\text { to PDF. You must view these files (e.g. movies) online. }\end{array}$ \\
\hline \begin{tabular}{l} 
files.zip \\
\hline
\end{tabular}
\end{tabular}

\section{s scholarONE" \\ Manuscript Central}




\title{
RESEARCH ARTICLE
}

\section{Submillimetre-wave spectrum of diacetylene and diacetylene- $d_{2}$}

\author{
Luca Bizzocchi $^{a, *}$, Claudio Degli Esposti ${ }^{b}$, and Luca Dore ${ }^{b}$ \\ ${ }^{a}$ CAAUL, Observatório Astronómico de Lisboa, Tapada da Ajuda, 1349-018, Lisboa \\ (Portugal); ${ }^{b}$ Dipartimento di Chimica "G. Ciamician", Università di Bologna, \\ via F. Selmi 2, 40126 Bologna (Italy)
}

(29 January 2010)

\begin{abstract}
Rovibrational bands between closely lying bending vibrational states of normal diacetylene and its bideuterated variant have been recorded in the submillimetre-wave region using a source-modulation spectrometer. The two isotopologues were produced by pyrolysis $\left(1200^{\circ} \mathrm{C}\right)$ of benzene or benzene- $\mathrm{d}_{6}$ vapour in a flow reactor. Measurements of the $\nu_{8}-\nu_{6}$ band and of its $\nu_{9}$-associated hot band have been extended up to $615 \mathrm{GHz}$ and $420 \mathrm{GHz}$, respectively. Rotational and vibrational $l$-type resonance were taken into account in the analysis of the $\nu_{8}+\nu_{9}-\nu_{6}-\nu_{9}$ band; in addition the cubic anharmonic resonance existing between the $v_{8}=v_{9}=1$ state and the $v_{3}=1$ stretching state has been considered explicitly in the least-squares fits to the observed frequencies. Very accurate values of the band origins and of numerous spectroscopic constants have been determined for both isotopologues.
\end{abstract}

Keywords: Vibration rotation bands; Diacetylene; Hot bands; Pyrolysis; Resonances

\section{Introduction}

Diacetylene $(\mathrm{H}-\mathrm{C} \equiv \mathrm{C}-\mathrm{C} \equiv \mathrm{C}-\mathrm{H})$ and its bideuterated isotopologue are symmetric linear molecules without permanent electric dipole moment. Notwithstanding this, it has been established that the molecular vibrations through the $v_{6}$ and $v_{8}$ normal modes, involving the two apical $\mathrm{C}-\mathrm{H}$ bendings, produce an appreciable vibrationally induced dipole moment $[1,2]$. The magnitude of this effect is quite consistent upon $\mathrm{H}-\mathrm{D}$ isotopic substitution: it accounts for the small ground-state dipole moment of the asymmetric $d_{1}$ isotopologue that allowed the observation of its pure rotational spectrum [3-6]. More interestingly, it also produces large dipole moment changes with the excitation of $v_{6}$ and $v_{8}$ bending vibrations, and thus enhanced transition moments for the overtone, combination, and difference bands associated with these quanta. This phenomenon is common to many acetylene-type molecules and is caused by the large values of the second dipole moment derivatives with respect to the $\mathrm{C} \equiv \mathrm{C}-\mathrm{H}$ bending curvilinear coordinates $[3,7-9]$.

Normal diacetylene was the subject in the past of extensive high-resolution infrared investigations (see Refs. [10, 11] and references therein), whereas the $d_{2}$-variant was less studied and its vibrational fundamentals are known from low-resolution IR and liquid-phase Raman measurements [12]. In symmetric diacetylenes, the vibrational states $v_{6}=1$ and $v_{8}=1$ are located at ca. $630 \mathrm{~cm}^{-1}$

*Corresponding author. Email: bizzocchi@oal.ul.pt 
for the normal isotopologue, and at ca. $500 \mathrm{~cm}^{-1}$ for the $d_{2}$ variant; in both cases, the energy difference between them is few wavenumber units. The vibrational band $\nu_{8}-\nu_{6}$ has a band origin in the millimetre-wave (mm-wave) region below $100 \mathrm{GHz}$ and, though forbidden, due to the enhanced transition moment it is well suited to be investigated with mm-wave spectroscopy. Differently, the analogous $\nu_{5}-\nu_{4}$ band of the simpler acetylene molecule is placed much more higher in frequency $\left(\nu_{0} \sim 3.5 \mathrm{THz}[\mathbf{1 3}]\right)$ due to the larger separation between the relevant levels.

Earlier MW-spectroscopy studies on dyacetylene were performed by Tanaka and coworkers [14-16] who recorded the rotation vibration spectra of the $\nu_{8}-\nu_{6}$ band, and that of the $\nu_{9}$ associated hot band, in the $20-170 \mathrm{GHz}$ frequency interval. Nowadays, their spectral analyses provide the sole available experimental data set for the mm-wave spectrum of symmetric diacetylenes, and its quality cannot be regarded as fully satisfactory due to the limited frequency and $J$ coverage, and also because of the incomplete rovibrational treatment of the hot band systems.

In this work we have extended to the submillimetre-wave (submm-wave) region the investigation of the $\nu_{8}-\nu_{6}$ band of $\mathrm{HC}_{4} \mathrm{H}$ and of the $\nu_{6}-\nu_{8}$ band of $\mathrm{DC}_{4} \mathrm{D}$, covering the $85-615 \mathrm{GHz}$ frequency interval and reaching a $J$ quantum number as high as 78. The $\nu_{9}$ associated hot bands have also been recorded up to a frequency of $420 \mathrm{GHz}$. Rotational and vibrational $l$-type resonances have been taken into account in the analyses of the hot band spectra and, in addition, the cubic anharmonic interaction between the $v_{8}=v_{9}=1$ bending combination state and the $v_{3}=1$ stretching state has been explicitly included in the least-squares fit to the observed transition frequencies. This analysis was supported by the results of recent high-level $a b$ intio calculations $[17,18]$, which provided estimates of a large set of spectroscopic parameters derived from CCSD(T) complete cubic and semi-diagonal quartic force fields. Reliable and very accurate band origins and spectroscopic parameters were obtained for each investigated band.

It should be pointed out that diacetylene, being the simplest polyyne, is an important molecule in many astrophysical environments. In the interstellar medium, the members of this family (together with cyanopolyynes) are thought to represent the main link between the observed gas phase species and the carbonaceous material responsible for the emission of the unidentified infrared bands (UIBs, see for example Refs. $[19,20])$. Also, they are likely to be important constituents of the C-rich planetary atmospheres, where complex hydrocarbons and nitriles form from the photolytic and catalytic dissociation of $\mathrm{CH}_{4}$ and $\mathrm{N}_{2}[21,22]$. Unfortunately, the search for polyynes in extraterrestrial sources is frustrated by the absence of a permanent dipole moment, which precludes their detection in the radio domain. To date very few mid-infrared astronomical detections of diacetylene have been reported: e.g. in the proto-planetary nebula CRL 618 [20] and in Titan's atmosphere [23].

The situation is likely to change in the near future, since the forthcoming ALMA facility [24] will bring a revolution in astronomical spectroscopy at millimetre/submillimetre wavelengths, opening new perspective in the planetary research. ALMA will resolve the planetary disks down to scales of $100 \mathrm{~km}$ : its increased sensitivity and the ability to isolate planetary limbs (thereby increasing the line-of-sight) will allow a search for weaker spectral features and the detection of many new compounds [25]. In this context, diacetylene is a promising target as, in hot regions of planetary atmospheres, its submm-wave vibration-rotation spectrum might be strong enough to allow detection. 


\section{Experimental details}

In the previous spectroscopic studies of Tanaka et al. [14-16] normal and bideuterated diacetylenes were prepared by chemical synthesis. In the present investigation diacetylene has been generated by gas phase pyrolysis of benzene (or esadeuterated benzene for $\mathrm{DC}_{4} \mathrm{D}$ ) at $1200^{\circ} \mathrm{C}$. The same pyrolysis apparatus previously used to produce other carbon chains (see for example Ref. [26]) was also employed to generate diacetylene. The precursors were flowed at a pressure of ca. 500 mTorr $(\sim 67 \mathrm{~Pa})$ through a quartz tube at $1200^{\circ} \mathrm{C}$, which was directly connected to the absorption cell of the mm-wave spectrometer, through which the reaction products were continuously pumped.

Though very weak, signals of the $\nu_{8}-\nu_{6}$ band of $\mathrm{HC}_{4} \mathrm{H}$ could be observed during the pyrolysis process and were used to optimise to the maximum extent the reaction conditions. The spectral measurements were then performed employing a purified sample. The crude products of the pyrolysis reaction were first collected in a large liquid- $\mathrm{N}_{2}$-cooled trap placed between the end of the absorption cell and the pumping system, and then transferred into a sample holder by low-temperature trap-to-trap distillation. The sample holder was then placed in a acetone slush bath at a the temperature of $-96.5^{\circ} \mathrm{C}$ while pumping continuously over it; this allow the nearly complete removal of the more volatile side-products. The remaining material was then allowed to warm until $-70^{\circ} \mathrm{C}$ and the gaseous fraction, very rich in diacetylene, was readily transferred in a storing sample holder. This material was permanently stored at $-25^{\circ} \mathrm{C}$ in a refrigerator, and was used to record the spectra at a static pressure of ca. $10 \mathrm{mTorr}(\sim 1.3 \mathrm{~Pa})$, renewing the sample in the cell twice a day.

The rovibrational spectra of $\mathrm{HC}_{4} \mathrm{H}$ and $\mathrm{DC}_{4} \mathrm{D}$ were observed in selected frequency regions between 85 and $615 \mathrm{GHz}$ using a source modulation mm-wave spectrometer, which employs four Gunn oscillators (Farran and Carlstrom) as main radiation sources to cover the fundamental frequency range $53-115 \mathrm{GHz}$. Higher frequencies were generated using three different frequency multipliers, one optimised for the $50-75 \mathrm{GHz}$ band, and a quadrupler and a sextupler operating in the $85-115 \mathrm{GHz}$ frequency interval. The oscillators were phase-locked to a suitable harmonic of the fundamental frequency emitted by a cm-wave solid state source (Wavetek), which was in turn locked to a computer-controlled frequency synthesiser (Schomandl). A Schottky-barrier diode (Millitech) and a liquid-helium-cooled InSb detector (QMC) were used to record the spectra below and above $200 \mathrm{GHz}$, respectively. The Gunn oscillators were frequency modulated at $16.7 \mathrm{kHz}$, and the detected signals were demodulated at $2 f$ by a lock-in amplifier, so that the second derivative of the actual spectrum profile was displayed by the computer-controlled acquisition system. The average precision of the frequency measurements was estimated to be $15 \mathrm{kHz}$.

\section{Observed spectra and analysis}

The peculiarities of the submm-wave spectra described in the present work deserve a brief discussion of the relevant energy levels, nuclear spin statistical weights and selection rules. Figure 1, based on the experimental data reported in the literature (see Ref. [18] and references therein), shows the vibrational energy levels of $\mathrm{HC}_{4} \mathrm{H}$ in the $600-900 \mathrm{~cm}^{-1}$ range above the ground state; each state is also labelled with the symmetry species spanned in the $D_{\infty h}$ point group. We observed mm-wave lines between the two singly excited bending states $v_{6}=1$ and $v_{8}=1$, and between the bending combinations $v_{6}=v_{9}=1$ and $v_{8}=v_{9}=1$ (indicated 
with the solid arrows in the plot). Due to the perpendicular nature of these vibration rotation bands, all three $P, R$, and $Q$ branches are present, and each $J$ transition is split into a multiplet, owing to $l$-type resonance effects between the different $l$ sublevels.

Symmetric diacetylenes have a set of equivalent hydrogen nuclei (H or D) and two sets of equivalent carbon nuclei which are interchanged by the $C_{2}$ symmetry operation. This produces different spin statistical weights according to the symmetry of the rovibrational wave function under the $C_{2}$ operation. Table 1 summarises the spin multiplicity for all the vibrational states of $\mathrm{HC}_{4} \mathrm{H}$ and $\mathrm{DC}_{4} \mathrm{D}$ involved in the investigated bands.

The spectra were analysed by expressing each rovibrational term using the formalism originally developed by Yamada and co-workers [27, 28], and employed previously to fit the excited state rotational spectra of several carbon chains (see for example Ref. [26]). Briefly, the rovibrational Hamiltonian is first represented using the symmetric top basis functions $\left|v_{6}^{l_{6}}, v_{8}^{l_{8}}, v_{9}^{l_{9}} ; J, k\right\rangle$, with the restriction $k=l_{6}+l_{8}+l_{9}$. Employing the simplified notation $\left|l_{6}, l_{8}, l_{9} ; k\right\rangle$ the elements of the Hamiltonian matrix which are diagonal in the quantum numbers $v_{t}$ and $l_{t}$ are:

$$
\begin{aligned}
\left\langle l_{6}, l_{8}, l_{9} ; k|\hat{H}| l_{6}, l_{8}, l_{9} ; k\right\rangle & =G_{v}+x_{L(69)} l_{6} l_{9}+x_{L(89)} l_{8} l_{9} \\
& +\left\{B_{v}+d_{J L(69)} l_{6} l_{9}+d_{J L(89)} l_{8} l_{9}\right\}\left\{J(J+1)-k^{2}\right\} \\
& -D_{v}\left\{J(J+1)-k^{2}\right\}^{2}+H_{v}\left\{J(J+1)-k^{2}\right\}^{3},
\end{aligned}
$$

where $G_{v}$ is the pure vibrational energy of the $\left|v_{6}, v_{8}, v_{9}\right\rangle$ state. The off-diagonal vibrational $l$-type doubling terms $\left(\Delta l_{t} \pm 2, \Delta l_{t}^{\prime} \mp 2, \Delta k=0\right)$ are given by the expression:

$$
\begin{aligned}
\left\langle l_{t} \pm\right. & \left.2, l_{t^{\prime}} \mp 2 ; k|\hat{H}| l_{t}, l_{t^{\prime}} ; k\right\rangle=\frac{1}{4}\left\{r_{t t^{\prime}}+r_{t t^{\prime} J} J(J+1)\right\} \\
& \times \sqrt{\left(v_{t} \mp l_{t}\right)\left(v_{t} \pm l_{t}+2\right)\left(v_{t^{\prime}} \mp l_{t^{\prime}}+2\right)\left(v_{t^{\prime}} \pm l_{t^{\prime}}\right)}
\end{aligned}
$$

while the off-diagonal rotational $l$-type doubling terms $\left(\Delta l_{t} \pm 2,|\Delta k|=2\right)$ have the general form:

$$
\begin{aligned}
\left\langle l_{t} \pm 2,\right. & \left.l_{t^{\prime}} ; k \pm 2|\hat{H}| l_{t}, l_{t^{\prime}} ; k\right\rangle=\frac{1}{4}\left\{q_{t}+q_{t J} J(J+1)\right\} \\
& \times \sqrt{\left(v_{t} \mp l_{t}\right)\left(v_{t} \pm l_{t}+2\right)} \\
& \times \sqrt{[J(J+1)-k(k \pm 1)][J(J+1)-(k \pm 1)(k \pm 2)]}
\end{aligned}
$$

The resulting energy matrix is factorised into symmetric and antisymmetric blocks adopting Wang-type linear combinations of wavefunctions [27]. Any sublevel belonging to a given vibrational state can be labelled through its $k$ value and by the ' + ' or ' -' superscripts which designate the sign of the symmetrised linear combination or, alternatively, with the $e, f$ parity label [29] using the correspondence $+\rightarrow e,-\rightarrow f$ for even $k$ and vice versa for odd $k$.

As mentioned above, the anharmonic interaction between the vibrational states $v_{3}=1$ and $v_{8}=v_{9}=1$, arising from the normal coordinate force constant $\phi_{389}$, has been considered in the present spectral analysis. The corresponding off-diagonal 
matrix elements in the symmetrized basis has the expression [30]:

$$
\begin{aligned}
e^{\left\langle 1,0^{0}, 0^{0} ; J, 0\right|} \hat{H}_{30}\left|0,1^{1}, 1^{-1} ; J, 0\right\rangle_{e} \\
\\
=W_{389}+W_{389 J} J(J+1)=\frac{1}{2} \phi_{389}+W_{389 J} J(J+1) .
\end{aligned}
$$

Over 200 new transition frequencies have been measured, and the complete list of analysed data is available as supplementary material. In the following subsections some details, dealing with the analyses performed for the various bands investigated, are given.

\section{1. $\nu_{8}-\nu_{6}$ band of $\mathrm{HC}_{4} \mathrm{H}$ and $\nu_{6}-\nu_{8}$ band of $\mathrm{DC} C_{4} \mathrm{D}$}

The $v_{6}=1$ and the $v_{8}=1$ bending fundamentals are close lying states; their energy difference, $\left|G_{6}-G_{8}\right|$, is ca. $3 \mathrm{~cm}^{-1}$ in both isotopologues, but in $\mathrm{DC}_{4} \mathrm{D}$ the position is inverted, i.e. $v_{8}=1$ lies at lower energy. Rovibrational transitions occur between states with the same overall parity, thus for $P$ and $R$ lines $(\Delta J= \pm 1)$ having $g \leftrightarrow u, e \leftrightarrow e$ and $f \leftrightarrow f$ transitions are allowed, with spin statistical weights given by Table 1 .

Since the value of the rotational constant, $B_{v}$, is a significant fraction of the vibrational energy separation, the rovibrational levels cross around $P(10)$ transition, the $P$ branch forms a band head and extends at frequency higher than band origin. This is well illustrated in Figure 2, which shows the calculated stick spectrum of the $\nu_{8}-\nu_{6}$ band of $\mathrm{HC}_{4} \mathrm{H}$ at $300 \mathrm{~K}$. It is apparent that both $P$ - and $R$-type transitions reach the maximum intensity well into the submm-wave region.

These bands had been previously investigated in the $16-168 \mathrm{GHz}$ frequency range by Tanaka and co-workers [14-16] who recorded 65 and 44 lines for $\mathrm{HC}_{4} \mathrm{H}$ and $\mathrm{DC}_{4} \mathrm{D}$, respectively. In the present work we extended the measurements into the submm-wave region, reaching maximum frequencies of $615 \mathrm{GHz}$ for $\mathrm{HC}_{4} \mathrm{H}$ and $550 \mathrm{GHz}$ for $\mathrm{DC}_{4} \mathrm{D}$. The $J$ interval covered by the measurements was considerably increased, having achieved transitions $R(59)$ and $P(77)$ for both isotopic variants. Some mm-wave lines, that exhibited large residuals in the previous analyses [15, 16], were also remeasured. The spectrum of the highest frequency line measured, $R(61)^{f}$ of $\mathrm{HC}_{4} \mathrm{H}$ is presented in Figure 3 .

The analysis of the spectra were performed through eqs. (1) and (3) expressing the band origin, $\nu_{0}$, as $G_{8}-G_{6}$ for $\mathrm{HC}_{4} \mathrm{H}$ and $G_{6}-G_{8}$ for $\mathrm{DC}_{4} \mathrm{D}$. All the previous mm-wave lines were included in the data sets with a reduced weighting factor $(w=$ $\left.1 / \sigma^{2}\right)$, in order to take into account their lower measurement precision. Separate fits, performed on homogeneous datasets, allowed to estimate average uncertainties of $\sigma=15 \mathrm{kHz}$ and $\sigma=40 \mathrm{kHz}$ for the present measurements and for the literature data $[14,15]$, respectively. The least-squares fits yielded much improved values of the band origins, rotational constants $B_{v}$, quartic centrifugal distortion constants $D_{v}$, and $l$-type doubling constants $q_{t}$. Furthermore, new determinations of the sextic distortion constants $H_{v}$ and of the centrifugal $l$-type parameters $q_{t J}$ were attained. The results are collected in Table 2. They exhibit a good agreement with those previously determined by Matsumura and Tanaka using more limited datasets [14, 15], as they are generally coincident within the quoted uncertainties. 


\section{2. $\nu_{8}+\nu_{9}-\nu_{6}-\nu_{9}$ hot band of $\mathrm{HC}_{4} \mathrm{H}$}

The bending combination states $\nu_{8}+\nu_{9}$ and $\nu_{6}+\nu_{9}$ are placed around $850 \mathrm{~cm}^{-1}$ in diacetylene [10]; as for the the singly excited states $v_{6}=1$ and $v_{8}=1$, the energy difference between them is ca. $3 \mathrm{~cm}^{-1}$, with the $\nu_{6}=\nu_{9}=1$ being the lower level. The $v_{9}=1$ state is a $\Pi_{u}$ species, thus the combination with $v_{6}=1\left(\Pi_{u}\right)$ give rise to three substates: $\Sigma_{g}^{+}$and $\Sigma_{g}^{-}$having $k=0$, and the doubly degenerate $\Delta_{g}$ with $|k|=2$. Analogously, the $v_{8}=v_{9}=1$ state consists of $\Sigma_{u}^{+}, \Sigma_{u}^{-}$, and $\Delta_{u}$ substates. The $\Sigma^{+}$and $\Sigma^{+}$states belongs to the species $e$ and $f$, respectively, while the $\Delta$ substates are split by rovibrational $l$-type resonances into $e, f$ doublets. $P$ - and $R$-type transitions of this band obey selection rules $e \leftrightarrow e$ and $f \leftrightarrow f$, with spin statistical weights given by Table 1 . The subband structure of the $\nu_{8}+\nu_{9}-\nu_{6}-\nu_{9}$ of $\mathrm{HC}_{4} \mathrm{H}$ is illustrated in Figure 4.

Previous mm-wave measurements had been already performed by Matsumura et al. [15] in the $24-175 \mathrm{GHz}$ frequency range. They performed first a band by band analysis, then the $l$-type resonance effects were considered in a coupled rovibrational fit which gave separate sets of spectroscopic parameters for $\Sigma^{+}, \Sigma^{-}$ and $\Delta$ substate, because the model employed at that time did not allow to obtain a single set of band constants. These authors noticed that the band origin of the $\Sigma_{g}^{+}-\Sigma_{u}^{+}$subband was red-shifted by ca. $1 \mathrm{~cm}^{-1}$ with respect to the other subbands origins. Furthermore, they found that the effective rotational constants, $B$, for the $\Sigma_{g}^{+}\left(k=0^{e}\right)$ level of the $v_{8}=v_{9}=1$ state is considerably lower than expected, giving an anomalous value of $\alpha_{9},-11.7 \mathrm{MHz}$, whereas the effective $B$ constants of the other substates gave $\alpha_{9}$ values very close to the known experimental value of $-12.5 \mathrm{MHz}$ [31]. Both anomalies were attributed to an anharmonic interaction with the $v_{3}=1\left(\Sigma_{g}^{+}\right) \mathrm{C}-\mathrm{C}$ stretching state, ca. $26 \mathrm{~cm}^{-1}$ higher in energy, through the $\phi_{389}$ normal coordinate force constant. The resonance pushes down the $\Sigma_{g}^{+}$sublevels of $v_{8}=v_{9}=1$ state and produces the rotational mixing responsible for its lower effective $B$ value. However, no accurate analysis was performed, and only rough estimates of the resonance parameters, based on reasonable assumptions, were given in Ref. [15].

In the present work we have extended the measurements of the $\nu_{8}+\nu_{9}-\nu_{6}-\nu_{9}$ band into the submm-wave region, covering the $340-420 \mathrm{GHz}$ frequency interval, in which both $R$ and $P$ branches of the vibration rotation spectrum of diacetylene are close to their maximum intensity (see Figure 2). The search for the new submmwave transitions has been guided by the effective subband constants of Ref. [15]. Lines belonging to the symmetry species $f$ (i.e. those of $\Sigma^{-}$and $\Delta(f)$ subbands) were found in a straightforward way, while the $e$ symmetry transitions could not be assigned until a proper treatment of the anharmonic resonance was performed. In particular, the $\Sigma^{+}\left(k=0^{e}\right)$ lines showed displacements up to $1.2 \mathrm{GHz}$ from the corresponding position predicted using the effective spectroscopic constants of Ref. [15].

Fifty-five new transition frequencies reaching $R(37)$ and $P(55)$ were finally assigned. They were analysed together with the previous mm-wave measurements [15] using a model which treats simultaneous all the subbands and includes explicitly the anharmonic resonance with the $v_{3}=1$ vibrationally excited states. The same weighting scheme used for the $\nu_{8}-\nu_{6}$ band was adopted to account for the different precision of the previous measurements [15] and the new submm-wave lines. The rotational constant of the unobserved $v_{3}=1$ state has been evaluated from the theoretical value of the $\alpha_{3}$ parameter [18], and using the experimental ground state $B_{0}$ value determined in Ref. [31]. Both off-diagonal resonance parameters $W_{389}$ and $W_{389 J}$ (see eq. (4)) were found to be important in reproducing the rotational mixing caused by the anharmonic resonance, though they are 
totally correlated and cannot be simultaneously determined. Therefore, we decided to fix $W_{389}$ at the value derived from the $\boldsymbol{a b}$ initio $\phi_{389}$ normal coordinate cubic force constant [32], whereas $W_{389 J}$ was released in the least-squares fit.

The rovibrational energies were calculated through eqs. (1)-(4) and expressing the band origin, $\nu_{0}$ as the energy difference between the $v_{8}=v_{9}=1$ and the $v_{6}=v_{9}=1$ states. The fitted parameters for this band are reported in Table 3 . The spectroscopic parameters determined include: very accurate values for $B$ and $D$ of both states, the $l$-type doubling constants $q_{6}, q_{8}$, and $q_{9}$, and the vibrational parameters $x_{L(69)}, x_{L(89)}, r_{69}$, and $r_{89}$. The centrifugal corrections $r_{69 \mathrm{~J}}$ and $r_{89 \mathrm{~J}}$ could not be simultaneously determined, we thus kept $r_{89 J}$ constrained to zero and optimised $r_{69} \mathrm{~J}$ as this latter attained the best reduction of the fit residuals.

The unperturbed vibrational energy difference between the two interacting states, $v_{3}=1$ and $v_{8}=v_{9}=1, \Delta G_{v}$, could not be set free in the analysis due to large correlation between various fit parameters, then its value was optimised through a step-by-step procedure until a minimum of the residuals root mean square was achieved. The optimum fit value of $28.1 \mathrm{~cm}^{-1}$ compares favourably with the experimental one of $24 \mathrm{~cm}^{-1}$ [11].

\section{3. $\nu_{6}+\nu_{9}-\nu_{8}-\nu_{9}$ hot band of $D C_{4} D$}

In diacetylene- $d_{2}$, the bending combination states $v_{6}=v_{9}=1$ and $v_{8}=v_{9}=1$ are located at ca. $700 \mathrm{~cm}^{-1}$. As in the normal isotopologue, the energy difference between them is about $3 \mathrm{~cm}^{-1}$, but their positions are inverted, i.e. the $\nu_{8}=\nu_{9}=1$ state is placed at the lower energy. For the $\nu_{6}+\nu_{9}-\nu_{8}-\nu_{9}$ band, mm-wave measurements had been already performed by Matsumura et al. [16] in the 30$150 \mathrm{GHz}$ frequency interval.

We recorded further mm-wave transitions, reaching a maximum frequency of $205 \mathrm{GHz}$, with the aim of attaining a larger data set for the coupled rovibrational analysis. Using the band constants of Ref. [16], the identification of the 25 new lines was accomplished in a straightforward way. These frequencies were analysed together with the previous measurements adopting the same weighting scheme described in the previous subsections.

In $\mathrm{DC}_{4} \mathrm{D}$, the vibrational energy difference between the $v_{8}=v_{9}=1$ and the perturbing state $v_{3}=1$ is about $140 \mathrm{~cm}^{-1}$ [18], thus the resulting resonance effects on the $\Sigma_{g}^{+}$substate are expected to be much weaker. However, we found that this anharmonic interaction must be taken into account if one aims at obtaining a satisfactory fit of all subbands simultaneously. The $v_{3}=1$ state was thus included in the energy level calculation using spectroscopic parameters derived as following: the $B$ value was evaluated from the ground state $B_{0}$ of Ref. [33] and from the ab initio $\alpha_{3}$ constant [17], the centrifugal distortion constants $D$, was obtained by scaling the corresponding value of the normal isotopologue, and the $\phi_{389}$ normal coordinated force constant was taken from the $\operatorname{CCSD}(\mathrm{T})$ computed force field of $\mathrm{DC}_{4} \mathrm{D}[34]$.

The rovibrational energies were calculated using eqs. (1)-(4) and the band origin, $\nu_{0}$, is expresses as the energy difference between the $v_{6}=v_{9}=1$ and the $v_{8}=$ $v_{9}=1$ states. The fitted parameters for this band are reported in Table 4 , they include: accurate values for $B$ and $D$, the $l$-type doubling constants $q_{6}$ and $q_{9}$, and the vibrational parameters $x_{L(69)}, x_{L(89)}, r_{69}$, and $r_{89}$. The other $v_{6^{-}}$and $v_{8^{-}}$ related $l$-type doubling parameters were kept fixed at the values determined for the fundamental band, whereas the centrifugal correction parameters $q_{9 J}$ was estimated 
from the value of the most abundant isotopologue by applying the scaling factor $q_{9}\left(\mathrm{DC}_{4} \mathrm{D}\right) / q_{9}\left(\mathrm{HC}_{4} \mathrm{H}\right)$.

\section{Conclusion}

The present paper extends the study of the vibration rotation spectra of symmetric diacetylenes into the submm-wave region reaching a frequency as high as $615 \mathrm{GHz} . \mathrm{HC}_{4} \mathrm{H}$ and $\mathrm{DC}_{4} \mathrm{D}$ have been produced using a new method based on a high-temperature ring cleavage reaction of benzene. The bending combination bands $\nu_{8}-\nu_{6}$ of $\mathrm{HC}_{4} \mathrm{H}$ and $\nu_{6}-\nu_{8}$ of $\mathrm{DC}_{4} \mathrm{D}$ have been investigated over a wide frequency range, thus obtaining an improved set of spectroscopic parameters including the sextic centrifugal distortion constants $H$, which were determined for the first time.

New mm-wave and submm-wave measurements have been also performed for the $\nu_{9}$ associated hot bands, involving vibrationally excited state up to an energy of $850 \mathrm{~cm}^{-1}$. The rovibrational analyses of the extended data sets were performed using the linear molecule Hamiltonian developed by Yamada et al. [27], resulting in a single set of spectroscopic constants for each investigated band. The anharmonic resonance which couples the $v_{3}=1\left(\mathrm{C}-\mathrm{C}\right.$ stretch, $\left.\Sigma_{g}^{+}\right)$state with the $\Sigma_{g}^{+}\left(k=0^{e}\right)$ of the $v_{8}=v_{9}=1$ state has been explicitly taken into account in the least-squares fit to the observed frequencies, assuming the relevant spectroscopic parameters from recent high level theoretical calculations [17, 18].

Comparing the fit results of fundamental and hot bands, one can evaluate the value of the vibration rotation interaction constant, $\alpha_{9}$, from each pair of levels yielding: $-12.537 \mathrm{MHz}$ and $-12.523 \mathrm{MHz}$ for $\mathrm{HC}_{4} \mathrm{H}$, and $-10.103 \mathrm{MHz}$ and $-10.136 \mathrm{MHz}$ for $\mathrm{DC}_{4} \mathrm{D}$. These values compare nicely with the corresponding theoretical values [17] of $-12.56 \mathrm{MHz}$ and $-10.13 \mathrm{MHz}$, thus confirming the validity of the present spectral analysis.

The present work complements the recently published studies on the lowest bendings of acetylene and acetylene- $d_{2}[35,36]$, whose rovibrational transitions provide a mean for detection of these non-polar molecules with microwave precision. The vibrational energy difference between the $v_{5}=1\left(\Pi_{u}\right)$ and $v_{4}=1\left(\Pi_{g}\right)$ states is $117 \mathrm{~cm}^{-1}$ in $\mathbf{H C C H}$ and $27 \mathrm{~cm}^{-1}$ in DCCD, thus the $\nu_{5}-\nu_{4}$ difference bands occur in the terahertz and far infrared regions. In diacetylene and diacetylene- $d_{2}$, the energy difference between the close lying $v_{8}=1$ and the $v_{6}=1$ levels is $2.40 \mathrm{~cm}^{-1}$ and $-2.88 \mathrm{~cm}^{-1}$, respectively. The maximum intensity of the corresponding difference bands is thus placed in the $\mathrm{mm}$-wave region at any excitation temperature, making these molecules suitable targets for ground based observations.

\section{Acknowledgements}

Support from MIUR (PRIN 2007 funds, project "Trasferimenti di energia, carica e molecole in sistemi complessi") and from the University of Bologna (RFO funds) is gratefully acknowledged.

\section{References}

[1]K. Matsumura and T. Tanaka, J. Mol. Spectrosc. 116, 320 (1986). 
1

3

4

5

6

7

8

9

10

11

[2]K. Matsumura and T. Tanaka, J. Mol. Spectrosc. 116, 334 (1986).

[3]T. Etoh, K. Matsumura and T. Tanaka, J. Mol. Spectrosc. 89, 511 (1981).

[4] K. Tanaka, K. Kato and T. Tanaka, J. Mol. Spectrosc. 131, 272 (1988).

[5] O. Böttcher, N. Heineking, M. Andolfatto and D.H. Sutter, Z. Naturforsch. 44a, 89 (1989).

[6]K. Matsumura, R.D. Suenram, F.J. Lovas and T. Tanaka, J. Mol. Spectrosc. 240, 120 (2006).

[7]T. Tanaka, C. Yamada and K. Matsumura, J. Mol. Spectrosc. 63, 142 (1976).

[8] W.J. Lafferty, R.D. Suenram and D.R. Johnson, J. Mol. Spectrosc. 64, 147 (1977).

[9] K. Matsumura, T. Tanaka, Y. Endo and E. Hirota, J. Phys. Chem. 84, 1793 (1980).

[10] G. Guelachvili, A.M. Craig and D.A. Ramsay, J. Mol. Spectrosc. 105, 156 (1984).

[11]D. McNaughton and D.N. Bruget, J. Mol. Struct. 273, 11 (1992).

[12] N.L. Owen, C.H. Smith and G.A. Williams, J. Mol. Struct. 161, 33 (1987).

[13] Y. Kabbadj, M. Herman, G.D. Lonardo, L. Fusina and J.W.C. Johns, J. Mol. Spectrosc. 150, 535 (1991).

[14] K. Matsumura, T. Etoh and T. Tanaka, J. Mol. Spectrosc. 90, 106 (1981).

[15] K. Matsumura and T. Tanaka, J. Mol. Spectrosc. 96, 219 (1982).

[16] K. Matsumura and T. Tanaka, J. Mol. Spectrosc. 108, 299 (1984).

[17]S. Thorwirth, M.E. Harding, D. Muders and J. Gauss, J. Mol. Spectrosc. 251, 220 (2008).

18]A.C. Simmonett, H.F. Schaefer III and W.D. Allen, J. Chem. Phys. 130, 044301 (2009).

[19]P. Thaddeus and M.C. McCarthy, Spectrochim. Acta. A 57, 757 (2001).

[20] J. Cernicharo, A.M. Heras, A.G.G.M. Tielens, J.R. Pardo, F. Herpin, M. Guélin and L.B.F.M. Waters, Astrophys. J. 546, L123 (2001).

[21]J.H. Waite Jr., D.T. Young, T.E. Cravens, A.J. Coates, F.J. Crary, B. Magee and J. Westlake, Nature 316, 870 (2007).

[22]T. Fouchet, B. Bez'ard and T. Encrenaz, Space. Sci. Rev. 119, 123 (2005).

[23] T. Encrenaz, Plan. Space. Sci. 51, 89 (2003).

24] M. Tarenghi, Astrophys. Space Sci. 313, 1 (2008).

[25]E. Lellouch, Astrophys. Space Sci. 313, 175 (2008).

[26]L. Bizzocchi, C. Degli Esposti and P. Botschwina, Phys. Chem. Chem. Phys. 6, 46 (2004).

[27] K.M.T. Yamada, F.W. Birss and M.R. Aliev, J. Mol. Spectrosc. 112, 347 (1985).

[28] M. Niederhoff and K.M.T. Yamada, J. Mol. Spectrosc. 157, 182 (1993).

[29] J.M. Brown, J.T. Hougen, K.P. Huber, J.W.C. Johns, I. Kopp, H. Lefebvre-Brion, A.J. Merer, D.A. Ramsay, J. Rostas and R.N. Zare, J. Mol. Spectrosc. 55, 500 (1975).

[30] T. Okabayashi, K. Tanaka and T. Tanaka, J. Mol. Spectrosc. 195, 22 (1999).

[31]E. Arié and J.W.C. Johns, J. Mol. Spectrosc. 155, 195 (1992).

[32] S. Thorwirth, Private communication.

[33] R. Tay, G.F. Metha, F. Shanks and D. McNaughton, Struct. Chem. 6, 47 (1995).

[34] M.E. Harding, Private communication.

[35]S. Yu, B.J. Drouin, J.C. Pearson, H.M. Pickett, V. Lattanzi and A. Walters, Astrophys. J. 698, 2114 (2009).

[36] S. Yu, B.J. Drouin and J.C. Pearson, Astrophys. J. 705, 786 (2009). 


\section{Molecular Physics}

Table 1. Spin statistical weights of the rovibrational levels of $\mathrm{HC}_{4} \mathrm{H}$ and $\mathrm{DC}_{4} \mathrm{D}$.

\begin{tabular}{|c|c|c|c|c|c|c|c|}
\hline \multirow[b]{2}{*}{ State } & \multirow[b]{2}{*}{ Substate } & \multirow[b]{2}{*}{$|k|$} & \multirow[b]{2}{*}{ Species } & \multicolumn{2}{|c|}{$\mathrm{HC}_{4} \mathrm{H}$} & \multicolumn{2}{|c|}{$\mathrm{DC}_{4} \mathrm{D}$} \\
\hline & & & & even $J$ & odd $J$ & even $J$ & odd $J$ \\
\hline$v_{6}=1$ & $\Pi_{g}$ & 1 & $\left\{\begin{array}{l}e \\
f\end{array}\right.$ & 1 & 3 & 2 & 1 \\
\hline$v_{8}=1$ & $\Pi_{u}$ & 1 & $\left\{\begin{array}{l}e \\
f\end{array}\right.$ & $\begin{array}{l}3 \\
1\end{array}$ & $\begin{array}{l}1 \\
3\end{array}$ & $\begin{array}{l}1 \\
2\end{array}$ & $\begin{array}{l}2 \\
1\end{array}$ \\
\hline \multirow{3}{*}{$v_{6}=v_{9}=1$} & $\Sigma_{u}^{+}$ & 0 & $e$ & 3 & 1 & 1 & 2 \\
\hline & $\Sigma_{u}^{-}$ & 0 & $f$ & 1 & 3 & 2 & 1 \\
\hline & $\Delta_{u}$ & 2 & $\left\{\begin{array}{l}e \\
f\end{array}\right.$ & $\begin{array}{l}3 \\
1\end{array}$ & $\begin{array}{l}1 \\
3\end{array}$ & $\begin{array}{l}1 \\
2\end{array}$ & $\begin{array}{l}2 \\
1\end{array}$ \\
\hline \multirow{3}{*}{$v_{8}=v_{9}=1$} & $\Sigma_{g}^{+}$ & 0 & $e$ & 1 & 3 & 2 & 1 \\
\hline & $\Sigma_{g}^{-}$ & 0 & $f$ & 3 & 1 & 1 & 2 \\
\hline & $\Delta_{g}$ & 2 & $\left\{\begin{array}{l}e \\
f\end{array}\right.$ & $\begin{array}{l}1 \\
3\end{array}$ & $\begin{array}{l}3 \\
1\end{array}$ & $\begin{array}{l}2 \\
1\end{array}$ & $\begin{array}{l}1 \\
2\end{array}$ \\
\hline
\end{tabular}




\section{March 11, 2010 \\ Molecular Physics}

Molecular Physics

Table 2. Spectroscopic parameters determined for the $\nu_{8}-\nu_{6}$ band of $\mathrm{HC}_{4} \mathrm{H}$ and for the $\nu_{6}-\nu_{8}$ band of $\mathrm{DC}_{4} \mathrm{D}^{a}$.

\begin{tabular}{|c|c|c|c|c|}
\hline \multirow[b]{2}{*}{ Parameter } & \multicolumn{2}{|c|}{$\mathrm{HC}_{4} \mathrm{H}$} & \multicolumn{2}{|c|}{$\mathrm{DC}_{4} \mathrm{D}$} \\
\hline & $v_{6}=1$ & $v_{8}=1$ & $v_{6}=1$ & $v_{8}=1$ \\
\hline$/ \mathrm{MHz}$ & \multicolumn{2}{|c|}{$71868.3315(29)$} & \multicolumn{2}{|c|}{$86417.9502(51)$} \\
\hline$B_{v} \quad / \mathrm{MHz}$ & $4391.327832(74)$ & $4391.201952(73)$ & $3814.28534(11)$ & $3812.91731(11)$ \\
\hline$D_{v} \quad / \mathrm{kHz}$ & $0.472087(28)$ & $0.472112(27)$ & $0.333575(53)$ & $0.334047(50)$ \\
\hline$H_{v} \quad / \mathrm{mHz}$ & $0.0330(34)^{b}$ & $0.0330(34)^{b}$ & $0.0298(77)$ & $0.0334(70)$ \\
\hline / $\mathrm{MHz}$ & $2.485082(94)$ & $2.409507(88)$ & $2.28762(10)$ & $2.27713(10)$ \\
\hline$q_{t J} \quad / \mathrm{Hz}$ & $-1.188(14)$ & $-1.302(15)$ & $-0.872(16)$ & $-1.248(15)$ \\
\hline$\sigma_{w}^{c}$ & \multicolumn{2}{|l|}{0.755} & \multicolumn{2}{|c|}{0.673} \\
\hline no. of lines & \multicolumn{2}{|l|}{121} & \multicolumn{2}{|l|}{97} \\
\hline
\end{tabular}

${ }^{a}$ The quantity in parentheses is one standard deviation in units of the last quoted digit.

Fitted as a mean value for both states.

Weighted root mean square deviation of the fit. 


\section{Molecular Physics}

Table 3. Spectroscopic parameters determined for the $\nu_{8}+\nu_{9}-\nu_{6}-\nu_{9}$ hot band of $\mathrm{HC}_{4} \mathrm{H}^{a}$.

\begin{tabular}{|c|c|c|c|c|}
\hline \multicolumn{2}{|c|}{ Parameter } & $v_{6}=v_{9}=1$ & $v_{8}=v_{9}=1$ & $v_{3}=1$ \\
\hline$\nu_{0}$ & / MHz & \multicolumn{2}{|c|}{$80130.928(25)$} & \\
\hline$x_{L(t 9)}$ & / GHz & $15.9292(25)$ & $13.3719(24)$ & \\
\hline$r_{t 9}$ & / GHz & $0.3408(44)$ & $9.6168(44)$ & \\
\hline$r_{t 9 J}$ & $/ \mathrm{kHz}$ & $-6.129(63)$ & & \\
\hline$B_{v}$ & / MHz & $4403.86468(12)$ & $4403.72458(12)$ & \multirow[t]{2}{*}{$4379.9094^{b}$} \\
\hline$d_{J L(t 9)}$ & $/ \mathrm{kHz}$ & $-5.12(12)$ & $-14.38(13)$ & \\
\hline$D_{v}$ & $/ \mathrm{kHz}$ & $0.490455(46)$ & $0.490433(50)$ & \multirow[t]{2}{*}{$0.47^{c}$} \\
\hline$q_{t}$ & / MHz & $2.50157(59)$ & $2.44806(54)$ & \\
\hline$q_{t J}$ & $/ \mathrm{Hz}$ & $-1.188^{d}$ & $-1.302^{d}$ & \\
\hline$q_{9}$ & / $\mathrm{MHz}$ & $6.17893(76)$ & $6.19055(85)$ & \\
\hline$q_{9 J}$ & / Hz & $-16.04^{e}$ & $-16.04^{e}$ & \\
\hline$\Delta G_{v}^{f}$ & $/ \mathrm{cm}^{-1}$ & & $28.1^{g}$ & \\
\hline$W_{389}$ & $/ \mathrm{cm}^{-1}$ & & $6.746^{b}$ & \\
\hline$W_{389 J}$ & / $\mathrm{MHz}$ & & $-0.78907(28)$ & \\
\hline \multicolumn{2}{|l|}{$\sigma_{w}^{h}$} & \multicolumn{2}{|c|}{0.762} & \\
\hline \multicolumn{2}{|c|}{ no. of lines } & \multicolumn{2}{|r|}{158} & \\
\hline
\end{tabular}

a The quantity in parentheses is one standard deviation in units of the last quoted digit. Assumed value, see text.

Ground state value, from Ref. [31].

Fixed to the $\nu_{8}-\nu_{6}$ band value.

$v_{9}=2$ state value, from Ref. [31].

Vibrational energy difference between the $v_{3}=1$ and the $v_{8}=v_{9}=1$ states.

Optimised through step-by-step procedure.

Weighted root mean square deviation of the fit. 


\section{March 11, 2010
Paqe 13 of \\ Molecular Physics}

Molecular Physics

Table 4. Spectroscopic parameters determined for the $\nu_{6}+\nu_{9}-\nu_{8}-\nu_{9}$ hot band of $\mathrm{DC}_{4} \mathrm{D}^{a}$.

\begin{tabular}{|c|c|c|c|c|}
\hline \multicolumn{2}{|c|}{ Parameter } & $v_{6}=v_{9}=1$ & \multirow{2}{*}{$v_{8}=v_{9}=1$} & \multirow[t]{2}{*}{$v_{3}=1$} \\
\hline$\nu_{0}$ & $/ \mathrm{MHz}$ & $84723.340(16)$ & & \\
\hline$x_{L(t 9)}$ & / GHz & $15.2159(54)$ & \multirow{3}{*}{$\begin{array}{r}14.1230(54) \\
2.4263(93)\end{array}$} & \\
\hline$r_{t 9}$ & / GHz & $2.0423(93)$ & & \\
\hline$r_{t 9 J}$ & $/ \mathrm{kHz}$ & $-36.60(19)$ & & \\
\hline$B_{v}$ & / $\mathrm{MHz}$ & $3824.38760(32)$ & \multirow{2}{*}{$\begin{array}{c}3823.05282(35) \\
-8.12(36)\end{array}$} & $3801.9443^{b}$ \\
\hline$d_{J L(t 9)}$ & $/ \mathrm{kHz}$ & $-11.24(31)$ & & \\
\hline$D_{v}$ & $/ \mathrm{kHz}$ & $0.34785(67)$ & $0.34827(63)$ & $0.33^{b}$ \\
\hline$q_{t}$ & / $\mathrm{MHz}$ & $2.2914(14)$ & $2.27713^{c}$ & \\
\hline$q_{t, J}$ & / $\mathrm{Hz}$ & $-0.872^{c}$ & $-1.248^{c}$ & \\
\hline$q_{9}$ & / $\mathrm{MHz}$ & $5.0762(65)^{d}$ & $5.0762(65)^{d}$ & \\
\hline$q_{9 J}$ & / $\mathrm{Hz}$ & $-12.8^{e}$ & $-12.8^{e}$ & \\
\hline$\Delta G_{v}^{f}$ & $/ \mathrm{cm}^{-1}$ & & $140.0^{b}$ & \\
\hline$W_{389}$ & $/ \mathrm{cm}^{-1}$ & & $11.512^{b}$ & \\
\hline$W_{389 \mathrm{~J}}$ & / $\mathrm{MHz}$ & & \multirow[t]{2}{*}{$-0.6070(22)$} & \\
\hline$\sigma_{w}^{g}$ & & & & \\
\hline \multicolumn{2}{|c|}{ no. of lines } & & $\begin{array}{c}0.699 \\
94\end{array}$ & \\
\hline
\end{tabular}

$a$ The quantity in parentheses is one standard deviation in units of the last quoted digit Assumed value, see text.

Fixed to the $\nu_{6}-\nu_{8}$ band value.

Fitted as a mean value for both states.

Scaled value, see text.

Vibrational energy difference between the $v_{3}=1$ and the $v_{8}=v_{9}=1$ states.

$g$ Weighted root mean square deviation of the fit. 


\section{Molecular Physics}

Page 14 of 17

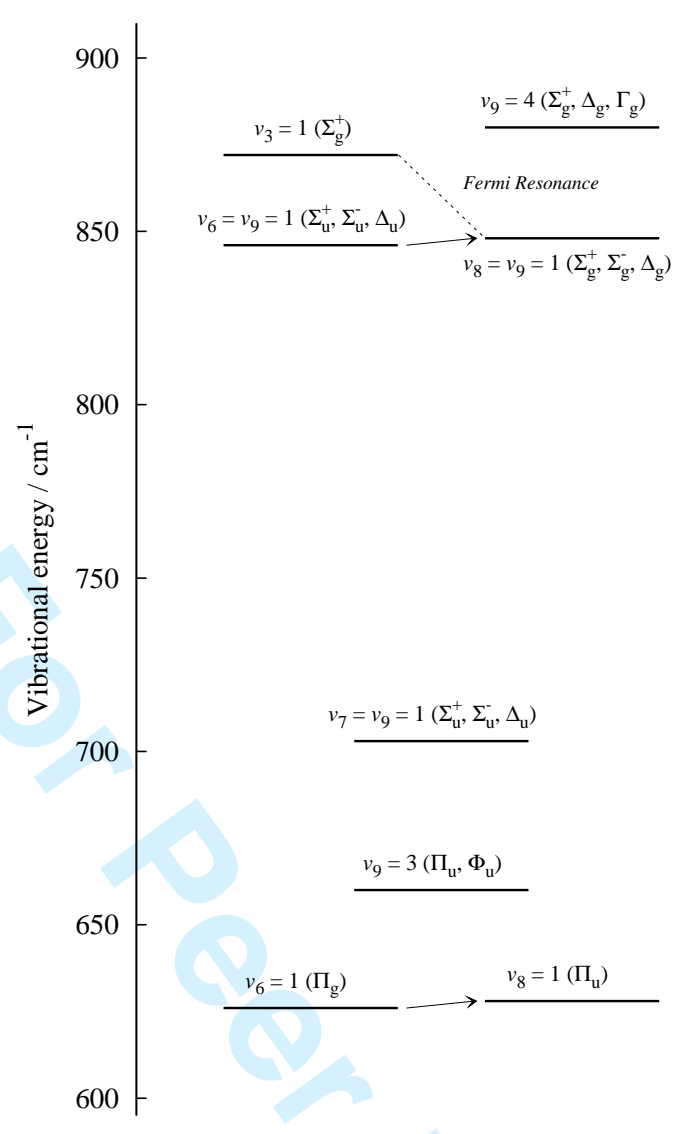

Figure 1. Vibrational states of diacetylene in the $600-900 \mathrm{~cm}^{-1}$ energy interval above the ground state (from Ref. [18]). The observed vibration rotation band are indicated by the arrows. The dotted line represents the anharmonic interaction considered in the present spectral analysis. 


\section{Page 15 of 17

4

5

8

9

10

11

12

13

14

15

16

17

18

19

20

21

22

23

24

25

26

27

28

29

30

31

32

33

34

35

36

37

38

39

40

41

42

43

44

45

46

47

48

49

50

51

52

53

54

55

56

57

58

59

60

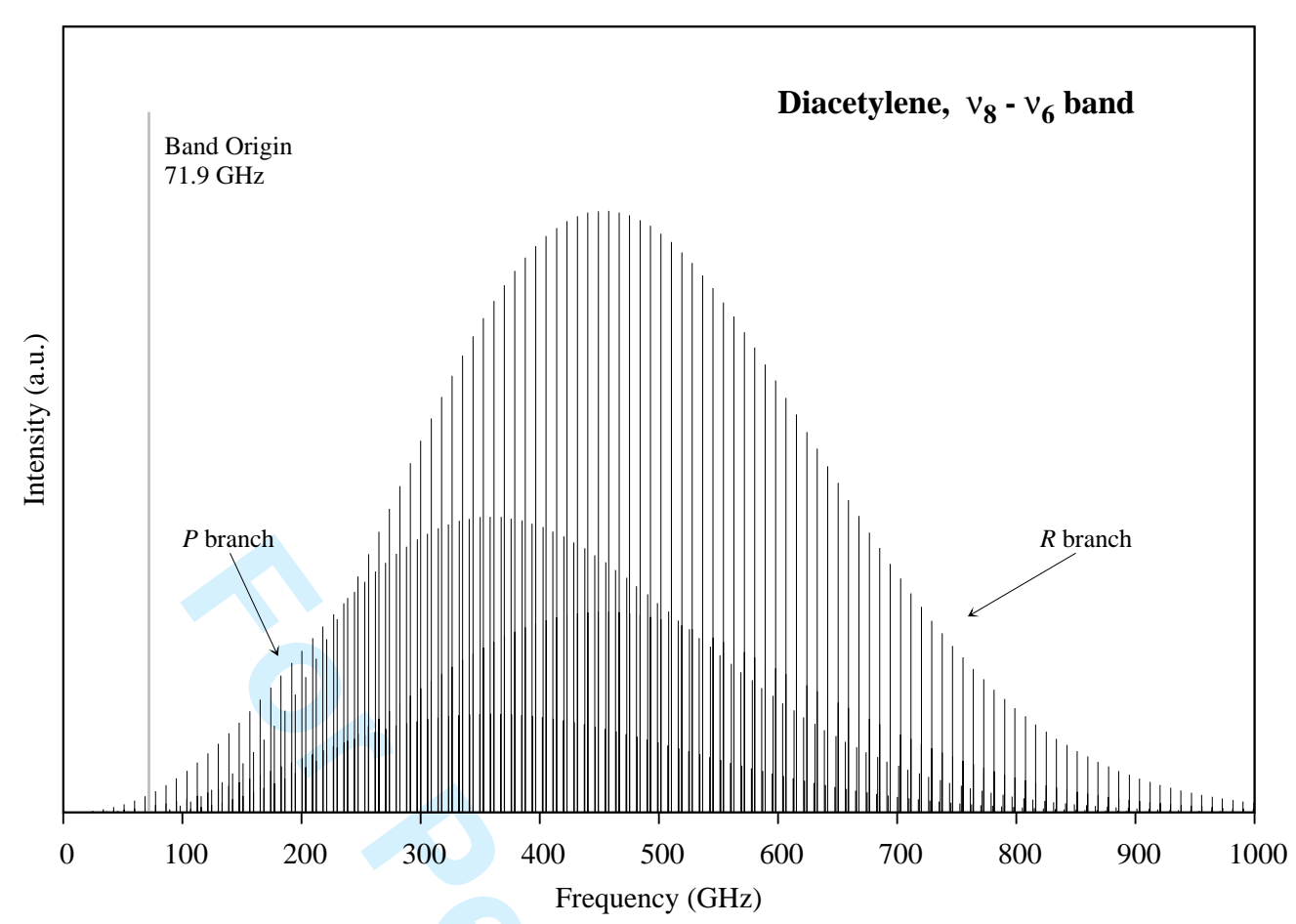

Figure 2. Calculated stick spectrum of the $\nu_{8}-\nu_{6}$ band of diacetylene at $300 \mathrm{~K}$. The apparent large line separations is due to the 3:1 intensity alternation due to spin statistical weights. The vertical grey stick at $71.9 \mathrm{GHz}$ indicates the band origin position. 


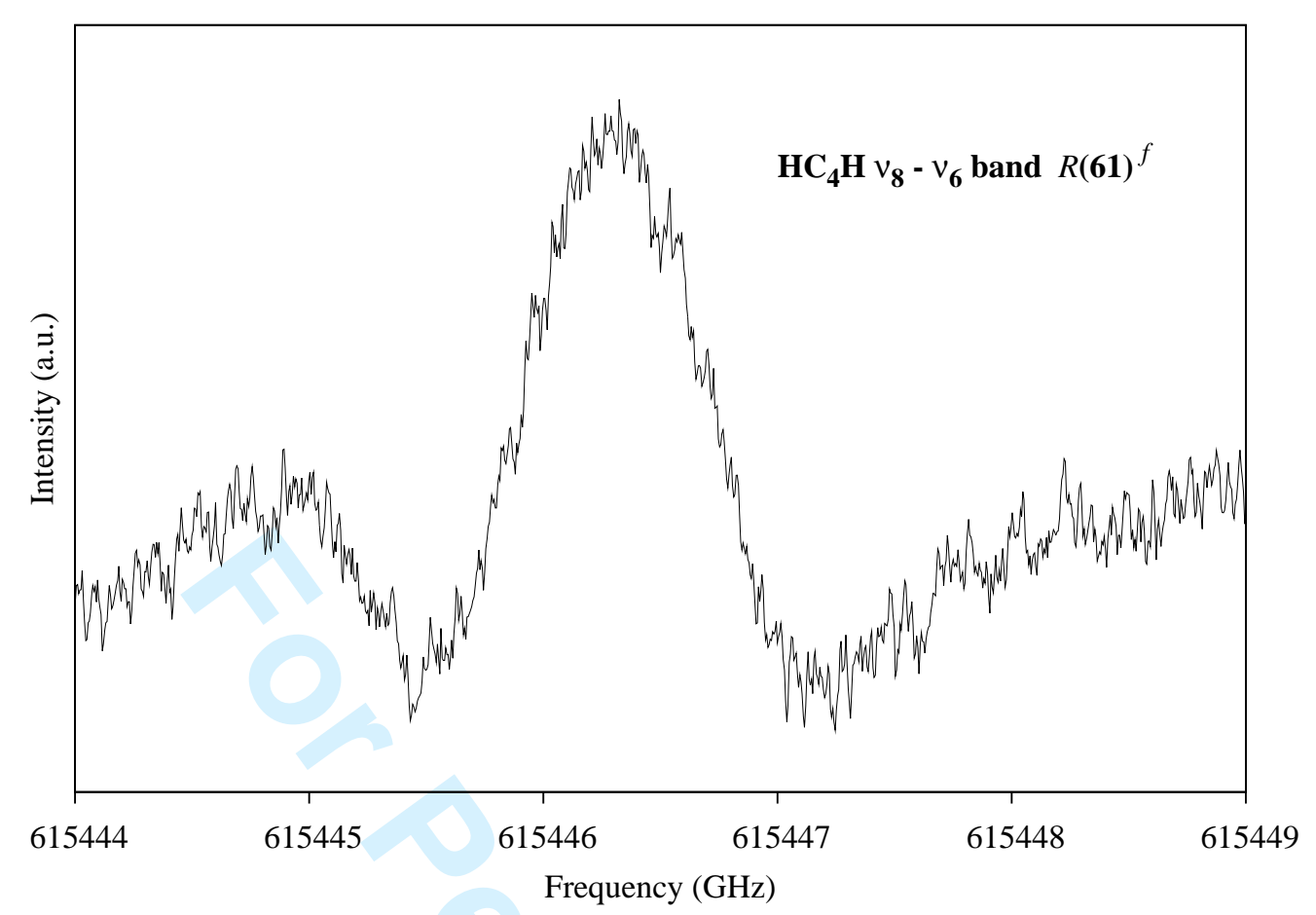

Figure 3. Spectrum of the $R(61)^{f}$ line of the $\nu_{8}-\nu_{6}$ band of diacetylene. Six scans with time constant of $0.3 \mathrm{~s}$ coadded (total integration time $180 \mathrm{~s}$ ). 
3

4

5

6

9

10

11

12

13

14

15

16

17

18

19

20

21

22

23

24

25

26

27

28

29

30

31

32

33

34

35

36

37

38

39

40

41

42

43

44

45

46

47

48

49

50

51

52

53

54

55

56

57

58

59

60

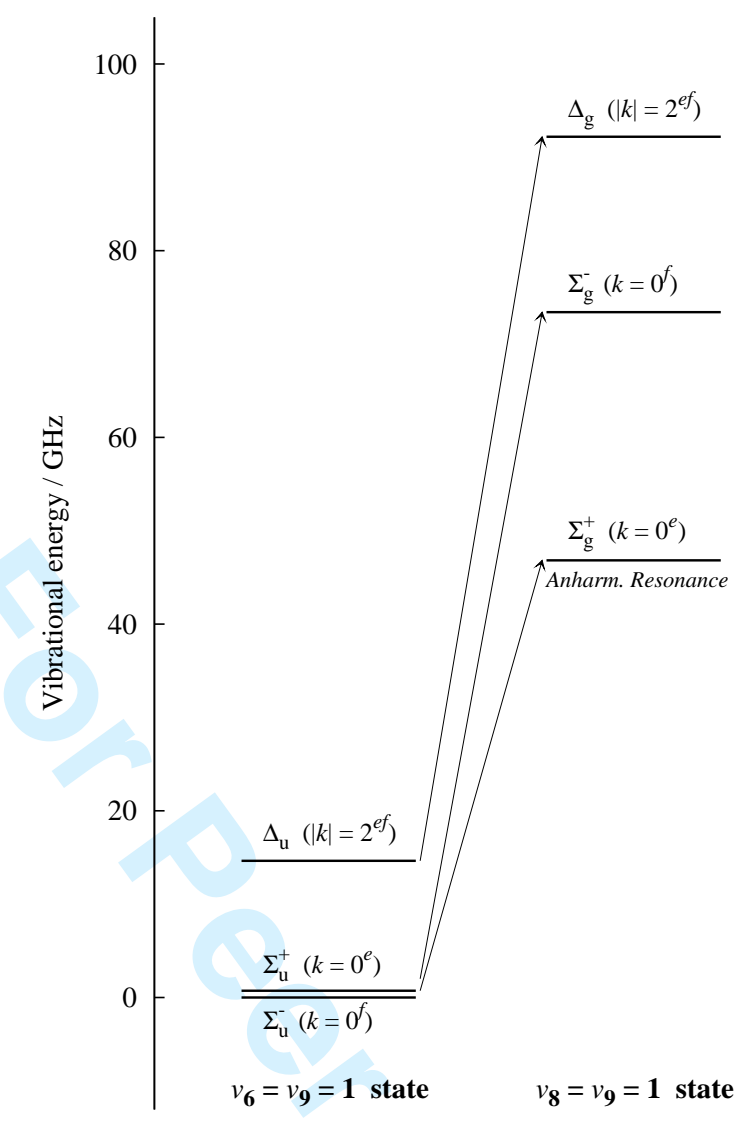

Figure 4. Subband structure of the $\nu_{8}+\nu_{9}-\nu_{6}-\nu_{9}$ band of diacetylene, calculated using the spectroscopic parameters of Table 3 . The arrows indicate the observed vibration rotation transitions. 